\title{
„Verschiedene Grade der Obszönität“: der Konsum von tierlichen Produkten außerhalb der Ernährung
}

\author{
Kurt Remele
}

Der südafrikanische, seit längerem in Australien lebende Schriftsteller J. M. Coetzee ist Literaturnobelpreisträger des Jahres 2003, zweifacher Gewinner des Booker-Preises und Fürsprecher der Tiere. In einem der seltenen Interviews, die er gibt, wurde er gefragt, ob er Vegetarier sei, und wenn ja, warum. Coetzee antwortete: „Ja, ich bin Vegetarier. Ich finde den Gedanken, Leichenteile in meinen Rachen zu stopfen, ziemlich widerwärtig und ich bin erstaunt, dass es so viele Menschen jeden Tag tun" (Henrik Engström/J. M. Coetzee 2004). ${ }^{1}$

Mag sein, dass es unklug ist, einen Beitrag über den Gebrauch von tierlichen Produkten außerhalb der Ernährung mit einer drastischen Äußerung über den Konsum von tierlichen Produkten in der Ernährung zu beginnen. Jonathan Safran Foer weist darauf hin, dass die meisten Menschen, die Tiere essen, nicht hören möchten, dass sie Leichen verspeisen. Sie bezeichneten deshalb den wissenschaftlich „unwiderlegbare[n] Satz ,Sie essen eine Leiche“ $[\ldots]$ als Übertreibung“. Nach Foer jedoch ist er „schlicht die Wahrheit" (Jonathan Safran Foer ${ }^{4} 2014,246$ ).

Wenn, wie Coetzee und Foer behaupten, Tiere nicht dazu da sind, dass wir Menschen sie verspeisen, ist es naheliegend, dass sie auch nicht dazu da sind, dass wir sie als Wintermäntel anziehen oder als Aktentaschen tragen. Es ist zu vermuten, dass zwischen dem Essen von Tieren und ihrem Gebrauch für Bekleidung und andere Zwecke ein Zusammenhang besteht: In beiden Fällen werden empfindungsfähige Lebewesen respektlos instrumentalisiert, in beiden Fällen werden ihnen unnötige Schmerzen zugefügt, in beiden Fällen werden sie getötet - dies alles, obwohl es in beiden Fällen in vielen Gegenden dieser Welt seriöse, wenn auch derzeit für viele noch ungewohnte Alternativen gibt.

Um diese Beziehung genauer zu betrachten, wenden wir uns wieder J. M. Coetzee zu. Er hat sich nicht nur in Interviews zu tierethischen Fragen geäußert, sondern ihnen sogar einen eigenen Roman gewidmet. Er be-

1 Diese und andere Übersetzungen aus englischsprachiger Originalliteratur stammen von Kurt Remele, dem Autor dieses Beitrages. 
titelte ihn schlicht mit „Das Leben der Tiere“. Das Buch ist kurz, geistreich und spannend zu lesen, zumindest für philosophisch einigermaßen Interessierte. Die Entstehung des Romans verdankt sich der Einladung der renommierten Princeton University an Coetzee, im Jahre 1997 die prestigeträchtigen „Tanner Lectures for Human Values“ zu halten. Anstelle der erwarteten Vorlesung trug Coetzee in Princeton jedoch zwei raffiniert konstruierte Kurzgeschichten vor, die später in dem Roman „Das Leben der Tiere“ zusammengeführt wurden. Die vorgetragenen Kurzgeschichten handelten von der australischen Schriftstellerin Elizabeth Costello. Diese war vom Appleton College, einer angesehenen Hochschule in einer USamerikanischen Kleinstadt, eingeladen worden, die bedeutenden GatesGastvorträge zu halten, die jedes Jahr einer anderen prominenten Person übertragen werden.

Frau Costello spricht in ihren Vorlesungen am Appleton College, wo ihr Sohn John Assistenzprofessor für Physik und Astronomie ist, nicht über ihr eigenes Leben oder ihre Romane, sondern über ein Problem, das sie persönlich seit längerem sehr beschäftigt, nämlich die Beziehung zwischen Menschen und Tieren, vor allem die menschliche Grausamkeit gegenüber Tieren. „Wenn ich zu Ihnen über Tiere spreche“, erklärt Costello in ihrem ersten Vortrag,

„will ich Sie mit der Schilderung der Schrecken ihres Lebens und Sterbens verschonen. Obschon ich keinen Grund zu der Annahme habe, dass Ihnen deutlich vor Augen steht, was Tieren in diesem Augenblick überall auf der Welt in Produktionsstätten (ich zögere, sie noch Bauernhöfe zu nennen), in Schlachthöfen, auf Fangschiffen, in Versuchslabors angetan wird. [...] Ich möchte Sie nur darauf hinweisen, dass diese Schrecken, die ich hier ausspare, trotzdem im Mittelpunkt dieser Vorlesung stehen“ (J. M. Coetzee 1999, 19).

Nach Costellos erster Rede findet ein feierliches Abendessen statt, zu dem Professor Garrard, der Rektor des College, eingeladen hat. Frau Costellos harsche Kritik am herrschenden Umgang mit Tieren hat im Kollegium der Professorinnen und Professoren Erstaunen, Verwirrung und Widerspruch ausgelöst. In der Tischkonversation kommt Garrard auf religiöse Speisevorschriften zu sprechen. Er regt damit eine lebhafte Diskussion über reine und unreine Nahrungsmittel an, über Schlachtvorschriften und über Sühnerituale, mit denen man schon in der Antike den Segen Gottes für das Schlachten und Essen von Tieren erbeten hat.

„Vielleicht ist das der Ursprung der Götter“, wirft Costello ein. „Vielleicht haben wir die Götter erfunden, damit wir sie verantwortlich machen können. Sie haben uns erlaubt, Fleisch zu essen. [...] Es ist nicht unsere 
Schuld, es ist die ihre. Wir sind nur ihre Kinder.“ „Und das glauben Sie?“, fragt die Frau des Rektors vorsichtig. Frau Costello antwortet mit einem Bibelzitat: „Und Gott sprach: Alles, was sich regt und lebt, das sei eure Speise.“ Und sie fügt hinzu: „Das ist bequem. Gott hat uns gesagt, dass es in Ordnung ist“(J. M. Coetzee 1999, 41).

Garrard versucht zu deeskalieren. Er bekennt, dass er "großen Respekt“ vor Costellos vegetarischer Ernährungsweise habe. „Ich trage Lederschuhe“, antwortet die Schriftstellerin darauf. „Ich trage eine Handtasche aus Leder. Ich würde an Ihrer Stelle nicht allzu viel Respekt haben.“ „Man kann doch zweifellos einen Unterschied machen zwischen dem Essen von Fleisch und dem Tragen von Leder“, wirft Garrard ein. „Unterschiedliche Grade von Obszönität“ (J. M. Coetzee 1999, 43-44), antwortet Costello lapidar.

\section{Streitgespräch: Die Schriftstellerin und der theologische Ethiker}

Was wäre, wenn man dem fiktiven Kollegium der Professorinnen und Professoren des Appleton College einen fiktiven theologischen Ethiker hinzufügte? Die Einführung einer solchen imaginären Figur in den vorliegenden Kontext erscheint mir reizvoll. Dieser Theologieprofessor würde Schuhe aus Rindsleder und einen Pullover aus Merinowolle tragen. In der kalten Jahreszeit würde er einen Trachtenjanker aus Schurwolle mit echten Hirschhornknöpfen anziehen oder eine Daunenjacke. Eine Wildledermütze mit Pelzbesatz aus Kaninchenfell zierte sein Haupt. Letztere würde er während der Lehrveranstaltungen in einer Aktentasche aus edlem Kalbsleder verstauen. ${ }^{2}$

Der Wissenschaftler würde ein hitziges Streitgespräch mit Elizabeth Costello über die ethische Beurteilung von Bekleidung führen. Er würde seine „tierproduktlastige“ Ausstattung mit dem Hinweis auf Schöpfungsordnung und Bibel rechtfertigen, auf die moraltheologische Tradition und auf den Weltkatechismus, der klar und deutlich feststellt, dass der Mensch das Recht habe, „sich der Tiere [...] zur Herstellung von Kleidern zu bedienen" (Katechismus der Katholischen Kirche 2003, Art. 2417). Der theologische Ethiker würde Frau Costello darüber belehren, dass es eine ontologi-

2 Um es unmissverständlich klar zu stellen: Ich kenne tatsächlich keinen einzigen theologischen Ethiker, der sich so kleidet wie der hier geschilderte. Seine Argumente für das Essen und den Gebrauch von Tieren sind allerdings solche, die man in theologischen Ethiken traditionellerweise findet. 
sche Differenz zwischen rational denkenden Menschen und triebgesteuerten Tieren gebe, dass wir nun einmal töten müssten, um überleben zu können und dass die Jagd notwendig sei, um Ökosysteme zu erhalten. Aus der Bibel sei kein allgemein verpflichtendes Verbot des Gebrauchs von Tieren zum Nutzen des Menschen abzuleiten, ganz im Gegenteil. Außerdem seien nicht nur Tiere, sondern auch Pflanzen sensible Wesen. Würde man Topfpflanzen eine Symphonie von Mozart vorspielen, würden sie wesentlich besser gedeihen. ${ }^{3}$ „Es stimmt“, würde der Theologe gegenüber Costello möglicherweise zugeben, „dass wir Tiere nicht ,unnötig' leiden lassen und nicht aus Spaß töten dürfen. Aber wenn es Menschen zugutekommt, ist sowohl die Inkaufnahme eines bestimmten Ausmaßes von tierischem Leid als auch die Tötung von Tieren ethisch gerechtfertigt." Das Tragen von Lederschuhen sei keine Obszönität, wie sie behaupte, ganz im Gegenteil: Wie würde es denn aussehen, wenn er auf der Uni mit ausgetretenen Plastiksandalen daherkäme? Die Lederproduktion sei unverzichtbar. Außerdem lebten wir nach dem paradiesischen Sündenfall, auch in der freien Natur gehe es grausam zu und die Tiere müssten um ihr Überleben kämpfen. Wir müssten realistisch sein, übertriebene Sentimentalität sei unangebracht.

„Haben Sie schon mal von der britischen Autorin Brigid Brophy gehört?", würde Elizabeth Costello dem Theologen antworten, nachdem sie ihm lange und geduldig zugehört hat. „Frau Brophy zu kennen, wäre wichtig für Sie, denn sie hat sich schon sehr früh, 1965, glaube ich, war es, über Tierrechte geäußert, einen Beitrag dazu in der ,Sunday Times' publiziert, der damals viel Aufsehen erregte (Brigid Brophy 1965). Brophy hat einmal gesagt: ,Immer dann, wenn Menschen sagen, wir dürfen nicht sentimental sein, kann man davon ausgehen, dass sie etwas Grausames vorhaben. Und wenn sie dann hinzufügen: Wir müssen realistisch sein, bedeutet das, dass sie Geld daraus machen wollen' (Brigid Brophy 1966).“

Costello würde sich räuspern, den Theologen ansehen und feststellen: „Das Problem ist, dass wir Menschen so vieles als ethisch berechtigten Gebrauch von Tieren hinstellen, was in Wahrheit eklatanter Missbrauch ist. Wir merken das nur nicht, wir nehmen nicht wahr, was wir nichtmenschlichen Tieren, die einen ,Eigenwert' (Franziskus 2015, Nr. 16, 33, 208) und eine,Würde' (Kurt Remele 22019) haben, am laufenden Band antun. Und was Artgrenzen und die klassische Unterscheidung zwischen Menschen

3 Bei dieser Behauptung handelt es sich um eine "moderne Sage“ (Englisch „urban myth" oder „urban legend“), eine unwahre Anekdote, die so oft erzählt wird, dass sie von vielen für wahr gehalten wird. 
und Tieren betrifft, sind diese nicht nur menschliche Konstruktionen, um unsere Ausbeutungspraxis zu rechtfertigen? Ist ein Schimpanse uns Menschen nicht wesentlich ähnlicher als einer Qualle oder einer Zecke? Warum bezeichnen wir dann sowohl Schimpansen als auch Quallen und Zecken als Tiere, uns aber als Menschen? Natürlich gibt es komplexe Fragen und moralische Konflikte, wo gibt es die nicht? Was das Quälen und Töten von Tieren für unsere Bekleidung betrifft, scheint mir das grundsätzliche Problem jedoch ganz simpel zu sein: Man ist, wenn ich mich recht erinnere, in der Ethik doch immer verpflichtet, dann, wenn es mehrere gleichwertige Wege gibt, ein bestimmtes Ziel zu erreichen, jenen zu wählen, der weniger gewaltsam ist und das Wohlergehen der Beteiligten besser berücksichtigt. Wäre es nicht weniger brutal und mit keinem wirklich nennenswerten Verzicht für den Menschen verbunden“, würde Elisabeth Costello sagen, „Schuhe aus anderen Materialien als Leder zu tragen, auch wenn das manchen Menschen geradezu absurd erschiene? Würde es nicht millionenfaches Leid und millionenfaches Töten und Sterben verhindern? Nicht nur die Herstellung von Leder, auch jene von Pelzen, Schafwollpullovern und Daunenjacken erzeugt doch ein ungeheures Ausmaß an Schmerz und Leid bei den davon betroffenen Tieren. Und bitte argumentieren Sie nicht mit dem naturwissenschaftlich unhaltbaren Argument einer Gleichheit von Tieren und Pflanzen. Das ist ein allzu billiger Trick, oder, wie Sie vielleicht sagen würden, eine allzu plumpe Werturteilserschleichung: Das Ausstechen eines Salatkopfes wird mit dem Abstechen eines Schweins gleichgesetzt und schon ist letzteres schwer in Ordnung. So einfach geht das in Ihrer theologischen Ethik." 4

Jetzt ließe sich Frau Costello nicht mehr einbremsen: „Und kommen Sie mir bitte nicht mit dem Hinweis auf die Bibel im Allgemeinen, Jesus Christus im Besonderen, der nichts gegen Fischfang, Mastkälber und sich ins Meer stürzende Schweine hatte. Weder die Autoren der Schriften des Ersten Testaments noch Jesus Christus besaßen irgendwelche Kenntnisse

4 Die Unhaltbarkeit des Vergleichs zwischen Salatköpfen und Schweinsköpfen wurde schon von Konrad Lorenz betont. In gleicher Weise stellt der österreichische Biologe und Fachbuchautor Clemens G. Arvay klar: „Salat zu pflücken und Möhren auszubuddeln ist nicht dasselbe wie einem Tier die Kehle durchzuschneiden. Daran können die seit Jahrzehnten in regelmäßigen Abständen auftretenden Medien-Hypes auf das ,geheime ${ }^{6}$ Leben der Pflanzen auch nichts ändern. So geheim ist dieses Leben übrigens gar nicht. Die sogenannte Pflanzensoziologie ist seit langem Teil der Biologie und das Sozialleben der Pflanzen sowie deren Kommunikation innerhalb eines Ökosystems seit Jahrzehnten erforscht. Und kein seriöser Biologe hat jemals die Notwendigkeit gesehen, die Unterscheidung zwischen Pflanze und Tier aufzuheben, das [...] wäre wirklich absurd“ (Clemens G. Arvay 2016, 95). 
der kognitiven Ethologie von heute oder haben jemals etwas von HumanAnimal Studies gehört. Leid und Leben von Tieren können uns doch nicht egal sein, mir sind sie es jedenfalls nicht. Doch auch ich, die ich von Ihnen als sentimentale Tierschützerin gebrandmarkt werde, lebe ethisch keineswegs konsequent: Ich trage Lederschuhe, obwohl es in vielen Teilen der Welt bereits zahlreiche Alternativen gibt: Schuhe aus Mesh oder Mikrofasern, Kork oder Baumwolle.“

Damit verlassen wir die um einen theologischen Ethiker erweiterte Erzählung über Elizabeth Costello. Wir wenden uns der Frage zu, welche anderen Tierprodukte neben Fleisch die Menschen weitgehend unhinterfragt verwenden, um bestimmte Grund- oder Luxusbedürfnisse zu befriedigen. Einige von ihnen, besonders jene, die dazu dienen, uns zu bekleiden, werden im Folgenden vorgestellt und aus tierethischer Sicht beurteilt.

\section{Die Haut abziehen: Leder}

Leder, die chemisch behandelte Haut von Tieren, wird von vielen als alternativloses und ökologisch vorteilhaftes Naturprodukt betrachtet und daher gerne und reinen Gewissens gekauft (David Clough 2019, 91-92; Stella McCartney 2015; Albert Schweitzer Stiftung für unsere Mitwelt 2014 a; Verein gegen Tierfabriken o. J. a). Doch die Herstellung von Leder für Schuhe, Gürtel, Mäntel, Jacken, Taschen, Geldbörsen, Sofas und Autositze erzeugt enormes Leid für Tiere und Menschen und horrenden Schaden für die Umwelt. Die Ledergewinnung ist kein Abfallprodukt der Fleischproduktion, sondern ein Nebenprodukt, durch das die Schlachtung der Tiere noch profitabler wird.

Mehr als achtzig Prozent der weltweiten Lederproduktion findet im Globalen Süden statt, vor allem in China und Vietnam, Indien und Bangladesch. Leder wird hauptsächlich aus den Häuten von Rindern, Schweinen und Schafen hergestellt, aber keineswegs ausschließlich. Auch Ziegen und Kängurus, Schlangen und Echsen, Haien und Elefanten, Krokodilen und Alligatoren wird die Haut für die Ledergewinnung abgezogen, mitunter bei lebendigem Leib: So hat die internationale Tierschutzorganisation PETA im Jahre 2016 die grausamen Zustände in einer Krokodilleder-Farm in Vietnam aufgedeckt. Die Tiere wurden dort in kleinen kahlen Gehegen zusammengepfercht gehalten und teilweise bei Bewusstsein gehäutet. Etwa zwei Millionen Kühe und Ochsen werden jedes Jahr für die Lederindustrie über hunderte Kilometer illegal aus Indien, wo Kühe traditionell als heilige Tiere gelten, nach Bangladesch geschmuggelt: 
„Der Transport dauert bis zu einer Woche, teils ohne Versorgung mit Wasser und Nahrung, auf LKWs und teilweise im Gehen. Brechen die erschöpften Tiere unterwegs zusammen und reagieren auch auf Gertenschläge nicht mehr, wird ihnen Chili in die Augen gerieben [...], damit sie vor Schmerzen wieder aufstehen und den Weg fortsetzen“ (Albert Schweitzer Stiftung für unsere Mitwelt 2014 a).

Um Tierhäute in Leder umzuwandeln, werden riesige Mengen an Chemikalien eingesetzt, die teils krebserregend sind und auch in Schuhen, Jacken und anderen Produkten noch nachgewiesen werden können. Vergiftet werden nicht nur die in den Gerbereien beschäftigten Arbeiterinnen und Arbeiter, darunter zahlreiche Minderjährige, sondern auch das Umland und die dort lebenden Menschen und Tiere. Im 2. Band seiner wegweisenden und umfassenden Tierethik-Monografie stellt der an der University of Chester lehrende methodistische Theologe Daniel Clough fest:

„Umweltgifte, die von Gerbereien freigesetzt werden, stellen eine massive ökologische Bedrohung dar - auch für freilebende Wildtiere und der Prozess erfordert beträchtliche Mengen an Wasser. Sogar im Vergleich mit synthetischen Alternativen aus Kunststoff, die von Öl abhängig sind, ist Leder in vielen ökologischen Bereichen wesentlich problematischer" (David Clough 2019, 92).

Wer Produkte aus Leder trägt oder verwendet, handelt nicht tierfreundlich. Er oder sie handelt auch nicht menschenfreundlich und nicht ökologisch. Der Wechsel zu alternativen Produkten ist ethisch geboten. Die Herstellung von Schuhen aus Naturmaterialien wie Hanf, Baumwolle oder Kork oder aus atmungsaktivem synthetischem Material kann zudem auch ökonomisch äußerst sinnvoll sein: In den ersten 3 Monaten des Jahres 2019 konnte die bekannte, vor allem bei jungen Leuten beliebte Schuhmarke Dr. Martens ihren Profit durch die Einführung veganer Schuhmodelle um siebzig Prozent steigern (Jasper Jolly 2019).

\section{Ins Fleisch schneiden: Wolle}

Schafen, deren Fell geschoren und zu Wolle verarbeitet wird, geht es zumindest auf den ersten Blick wesentlich besser als Rindern, die für die Produktion von Leder gequält und geschlachtet werden. Wirklich gut geht es ihnen in der Regel jedoch nicht. „Als , nachwachsender' Rohstoff hat Wolle bislang einen guten Ruf“, ist deshalb auf der Website der deutschen „Albert Schweitzer Stiftung“ zu lesen, „wir zeigen Ihnen, warum es dennoch 
sinnvoll ist, Wollprodukte zu meiden und auf alternative Fasern zurückzugreifen“ (Albert Schweitzer Stiftung für unsere Mitwelt 2016).

Welche Gründe werden von der Albert Schweitzer Stiftung und anderen Tierschutzorganisationen genannt, die Wolle zu einem tierethisch fragwürdigen Produkt machen? (Albert Schweitzer Stiftung für unsere Mitwelt 2016; David Clough 2019, 93; Vier Pfoten 2020.) Zunächst ist darauf hinzuweisen, dass Schafe nur deshalb geschoren werden müssen, weil sie auf die Ausbildung enormer Wollmassen hin gezüchtet wurden. Von Natur aus hat ein Schaf gerade so viel Wolle, wie er oder sie es braucht, um bei großer Kälte nicht zu frieren. In der globalen Wollproduktion werden Schafe jedoch fast ausschließlich als lebende Rohstofflieferanten betrachtet und entsprechend behandelt: Kurz nach der Geburt werden die Schwänze ohne Schmerzausschaltung kupiert, männliche Schafe werden betäubungslos kastriert. Können Schafe aus Altersgründen keine entsprechend große Wollmenge mehr liefern, werden sie zu Fleisch verarbeitet. In Australien, das zusammen mit Neuseeland und China zu den Hauptlieferanten von Schafwolle zählt, werden ,ausrangierte“ Schafe verschifft und in den Nahen Osten oder nach Nordafrika gebracht, dies häufig ohne ausreichend Nahrung und Zugang zu Wasser. Sterben die Tiere nicht an Bord, werden sie in den Ankunftsländern geschlachtet, meist durch Schächten und ohne Betäubung.

Das Scheren kann für Schafe belastend sein und sie zudem verletzen: „Bei dem Versuch, in möglichst kurzer Zeit möglichst viele Schafe zu scheren, entstehen oft auch große Schnittwunden, die zum Teil nur unachtsam und ohne Betäubung genäht werden“ (Albert Schweitzer Stiftung für unsere Mitwelt 2016). Merinoschafe besitzen zudem eine angezüchtete faltige Haut, um besonders viel Wolle zu produzieren. Weil sich in den Hautfalten der Tiere schnell Feuchtigkeit und am Hinterteil Kot und Urin ansammeln, legen Fliegen dort Eier ab. Die ausschlüpfenden Maden machen die Tiere krank. Um den Parasitenbefall zu verhindern, werden den zwei bis zehn Wochen alten Lämmern deshalb mit einer Metallschere große Hautfalten rund um After, Vulva und Schwanz herausgeschnitten. Diese Praxis wird „Mulesing“ genannt; sie wird ohne Betäubung und Schmerzstillung durchgeführt, die offenen Wunden werden nicht versorgt. „Mulesing“ hat zu weltweiten Protesten geführt, wird aber weiterhin umfangreich praktiziert.

David Clough weist darauf hin, dass es vereinzelt Schaffarmen gebe oder zumindest geben könnte - ob Clough hier im Indikativ oder Konjunktiv spricht, ist nicht ganz klar -, in denen die Tiere auf eine Art und Weise leben, die den Kriterien tierischen Wohlergehens entspricht und deren Wolle deshalb ethisch vertretbar sein mag: keine Kastration und kein 
Kupieren der Schwänze, zumindest nicht ohne Betäubung, kein „Mulesing“, vorsichtiges, behutsames Scheren ohne zeitlichen und ökonomischen Stress, ausreichende Weideflächen, keine Schlachtung nach vollbrachter mehrjähriger Arbeitsleistung (David Clough 2019, 100). Wollprodukte, die diesen Standards entsprechen, werden jedoch nicht leicht zu erhalten sein, und sie werden einen angemessenen Preis haben.

\section{Ausreißen:Daunen}

Als Füllstoff für Jacken und Mäntel, Bettdecken und Schlafsäcke sind Daunen sehr beliebt. Daunen stammen aus dem Untergefieder von Enten und Gänsen und besitzen im Unterschied zu Federn keinen Kiel. Sie zeichnen sich durch extreme Leichtigkeit und hohe Isolierfähigkeit aus (David Clough 2019, 97-98; Albert Schweitzer Stiftung für unsere Mitwelt 2014 b; Verein gegen Tierfabriken 2014; Daunenarten o. J.). Wie bei Wolle existiert laut David Clough auch bei Daunen lediglich eine einzige Variante, die tierethisch akzeptabel ist: das Sammeln von Daunen in nicht mehr gebrauchten Nestern. Vor allem in Norwegen und Island werden Entendaunen (Eiderdaunen) noch auf diese Art gewonnen: Nach der Brutzeit, wenn die Ente und die Jungvögel das Nest bereits verlassen haben, werden die Eiderdaunen in mühevoller Kleinarbeit von Hand aus Nestern gesammelt, bevor diese Wind und Wetter zum Opfer fallen, etwa 20 Gramm an Daunen pro Nest. Danach werden die Daunen von Hand gesäubert und mit Dampf gereinigt.

Daunen, die in Nestern gesammelt wurden, stellen allerdings die große Ausnahme dar. Fast immer werden die Daunen durch „Totrupf“ oder „Lebendrupf" gewonnen. Ersteres bezeichnet die Entfernung der Daunen von den Kadavern von Enten und Gänsen, die für die Fleischproduktion getötet wurden, letzteres das Ausreißen der Daunen aus der Haut von lebenden Tieren: Das kann maschinell erfolgen und zwar durch Maschinen, die ursprünglich für das Rupfen toter Tiere konstruiert wurden, oder mit der Hand. Beides ist für die Vögel extrem schmerzhaft:

„Die Tiere bleiben schwer verletzt und apathisch zurück. Die entstandenen Wunden werden ohne Betäubung vernäht, manchmal bleiben sie sogar unbehandelt. Diese äußerst qualvolle und schmerzhafte Prozedur müssen die Tiere mehrmals in ihrem Leben über sich ergehen lassen“ (Albert Schweitzer Stiftung für unsere Mitwelt 2014 b).

Es gibt heute zahlreiche natürliche und synthetische Daunenalternativen: Kapok etwa, die Hohlfaser des Kapokbaumes, oder diverse Kunstfasern. 


\section{Die Gliedmaßen brechen: Pelz}

Sowohl die Verwendung von Daunen als auch das Tragen von Pelz werden häufig damit gerechtfertigt, dass der Mensch andernfalls zu bestimmten Jahreszeiten oder in bestimmten Weltgegenden frieren oder erfrieren müsste. Dabei wird übersehen, dass es heute zu beiden tierlichen Produkten zahlreiche tierfreundliche Alternativen gibt. Pelz jedoch hat zum Unterschied von Daunen noch eine weitere Funktion: Pelz ist ein Luxusprodukt, mit dem der Träger oder die Trägerin die eigene Geltung und Bedeutung unterstreicht. Auch in der katholischen Kirche hat diese Form des "demonstrativen Konsums" (Thorstein Veblen [1899], 33-47) von Pelz eine lange Tradition. Papst Benedikt XVI. hat sie während seiner Amtszeit aufleben lassen.

Wenige Tage vor dem Weihnachtsfest des Jahres 2005 trug Papst Benedikt bei der Generalaudienz eine für obsolet gehaltene, nur außerhalb der Liturgie getragene päpstliche Kopfbedeckung, die ihm das Aussehen eines Weihnachtsmanns verlieh und dadurch einen beeindruckenden Anstieg seiner Medienpräsenz bescherte: den Camauro, eine aus rotem Samt gefertigte Wintermütze, die mit einem weißen Hermelinpelz verbrämt ist (Philippi Collection 2010 b).

In einem Interview, das der deutsche Journalist Peter Seewald einige Jahre danach mit ihm führte, hat Papst Benedikt das Aufsetzen des Camauro heruntergespielt: „Ich habe ihn nur einmal getragen. Mich hat einfach gefroren, und ich bin am Kopf empfindlich. Und ich habe gesagt, wenn wir schon den Camauro haben, dann setzen wir ihn auch auf" (Benedikt XVI. 2010, 110). Ich möchte dem Papst nicht gerne widersprechen, mag sein, dass es wirklich so war. Aber es bleiben Fragen: Wenn der Camauro neu für Benedikt angefertigt wurde, wie kann die Wahl dieser Kopfbedeckung dann eine völlig spontane Entscheidung gewesen sein? Wenn Papst Johannes XXIII. der letzte Papst war, der den Camauro vor Benedikt getragen hat, warum war die pelzumrandete Mütze dann für Benedikt nicht viel zu weit? Und hätte er nicht Monsignore Gänswein bitten können, eine Kopfbedeckung ohne Pelzrand zu finden. So etwas müsste es doch in den Kleiderkammern des Vatikans auch geben.

Der Camauro war zudem nicht das einzige pelzumrandete Kleidungsstück, das Benedikt zierte. Wiederholt trug er die päpstliche $\mathrm{Moz}(\mathrm{z})$ etta, einen Schulterumhang mit kleiner Kapuze, der sowohl in der Liturgie als auch außerhalb getragen werden kann. Die Mozzetta existiert in fünf Varianten, von denen drei mit Pelz verbrämt sind. Zwei dieser pelzbesetzten Mozzettas hat Benedikt getragen und damit wieder in die päpstliche Garderobe aufgenommen, nachdem Papst Johannes Paul II. sie bereits aussor- 
tiert hatte: die Winter-Mozzetta aus rotem Samt mit weißem Saum aus Hermelinfell und die Oster-Mozzetta aus weißer Damastseide, ebenfalls mit weißem Hermelinpelz verbrämt (Philippi Collection 2010 a).

\subsection{Der Schöne...}

In einem mit „Benedict, the best-dressed pope“ betitelten Beitrag in der Los Angeles Times im Februar 2013 stellte die erzkonservative Katholikin und renommierte Journalistin Charlotte Allen fest: „Als Papst Benedikt XVI. seinen bevorstehenden Amtsverzicht bekanntgab, war mein erster Gedanke kein religiöser. Er war vielmehr ausgesprochen oberflächlich. ,Das war's dann wohl mit dem bestgekleideten Pontifex aller Zeiten“" (Charlotte Allen 2013). „Der bestgekleidete Pontifex aller Zeiten“: Charlotte Allen zufolge hat Benedikt in den acht Jahren seines Pontifikats

„immer perfekt ausgesehen [...], ob er nun aufwändige liturgische Gewänder für die Osterliturgie trug oder eine einfache, aber perfekt geschneiderten weiße Soutane mit Schulterumhang [...] für den Alltag. Er war der ,Duke of Windsor der Päpste““ (Charlotte Allen 2013; vgl. Sarah Bailey 2013).

Falls Sie es nicht wissen sollten, der „Duke of Windsor“, der frühere König Edward VIII., wurde als einer der bestgekleideten Männer des 20. Jahrhunderts angesehen. Edward trat im Dezember 1936 von seinem Amt des Königs zurück, allerdings aus anderen Gründen als der bestgekleidete Papst knapp acht Jahrzehnte später.

Benedikts Vorliebe für „traditionellere, gar barocke Elemente päpstlicher Bekleidung“ (Henry Conway 2013) wurde nicht nur von konservativen Katholikinnen und Katholiken hochgeschätzt, sondern auch vom britischen Modejournalisten, Party-Promoter und Dandy Henry Conway. Er betitelte Benedikt anerkennend als „Protz-Papst“ (Henry Conway 2013) und untermauerte seine Wertschätzung mit der Geschichte über einen jungen Priester, der das Glück hatte, kurz vor einer Pontifikalmesse in der Sakristei des Petersdoms zugegen zu sein: „Der Papst kam mit dem Lift in die Sakristei herunter und konnte zwischen drei Garnituren an Gewändern und Mitren auswählen. Ohne Umschweife griff der Papst zu der prunkvollsten“ (Henry Conway 2013).

Menschen, die Luxus lieben, lieben in der Regel auch Pelze. In der Zeit, in der er das Papstamt ausübte, gehörte auch Joseph Ratzinger zu diesen Menschen. Die „bella figura“ des deutschen Pontifex hatte jedoch ihren 
Preis: Sie fügte einigen seiner nicht-menschlichen Mitgeschöpfe gewaltige Schmerzen zu und kostete sie das Leben.

\section{2 ... und das Biest}

„Unsere Schönheit ist unser Todesurteil“, hält das Hermelin den Menschen im Buch Caro uomo. Lettere degli animali (Lieber Mensch. Briefe der Tiere) des italienischen Franziskanermönchs Nazareno Fabbretti vor, in dem er sich in die Lage eines Löwen und eines Affen, eines Hundes und einer Katze, einer Hyäne und einer Biene und eben auch eines Hermelins versetzt und zu uns Menschen sprechen lässt. Das Hermelin beschwert sich darüber, dass Gewalt, Ehrgeiz und Selbstsucht der Menschen dazu geführt hätten, seine Artgenossen brutal zu fangen, zu züchten und zu töten:

„Ihr beraubt uns unserer Schönheit, um einen Markt für Luxusartikel zu schaffen, [nämlich] Pelzmäntel, die Monarchen, Prinzen, Päpsten gehören und den Frauen einiger der reichsten Männer dieser Erde. ... Dieser Diebstahl nährt den Stolz und die exhibitionistischen Tendenzen der Individuen und der egoistischen, privilegierten Klassen“ (Nazareno Fabbretti 1990, 45). ${ }^{5}$

Die allgemeine Tiervergessenheit des Christentums konkretisiert sich hier in der Hermelinvergessenheit Papst Benedikts XVI. Der Papst hat offenbar ausgeblendet, dass weltweit an die hundert Millionen Nerze, Füchse, Chinchillas, Waschbären, Wiesel, Hermeline und andere Pelztiere für die Pelzindustrie getötet werden. Dabei werden sie tierquälerisch in engen Käfigen gehalten: „Ihr kurzes Leben in Farmhaltungen ist eine Qual, voller Leiden und Schmerz. Sie haben keinen Platz, um elementarste, arttypische Verhaltensweisen auszuleben" (ProTier - Stiftung für Tierschutz und Ethik o. J.). Zwar sind Pelztierfarmen in einigen europäischen Ländern, darunter Österreich und Großbritannien, Bulgarien und Slowenien verbo-

5 Hier soll kurz auch die Verwendung von Seide zu menschlichen Bekleidungszwecken luxuriöser Art erwähnt werden. Bei der Herstellung von Seide werden riesige Mengen von Larven des künstlich gezüchteten, flugunfähigen Maulbeerspinners in ihren Kokons durch kochendes Wasser oder heißen Wasserdampf getötet. Da aber die Frage, ob und in welcher Weise diese Larven Schmerz empfinden können, naturwissenschaftlich weitgehend ungeklärt ist, wird die Herstellung von Seide hier nicht ausführlicher behandelt. Als Form der krassen Instrumentalisierung von Mitgeschöpfen ist die Seidenproduktion jedoch grundsätzlich kritisch zu bewerten (David Clough 2019, 98-99). 
ten, und zahlreiche Kaufhäuser bieten nur noch alternative Webpelze an. Doch es gibt auch einen neuen Trend zu edlen Pelzen als Statussymbol: „Es scheint, als könne man den Pelz nicht so leicht aus der Modewelt verbannen. Zu dick ist sein Fell. Pelz ist ein Lebensstil“ (Welt 2013). Zudem ist es in einigen Ländern, darunter Kanada und Russland, immer noch legal, für den Fang dieser Tiere monströse Fallen aus Metall, so genannte Tellereisen, zu verwenden. Diese schnappen so fest und rasch zu,

„dass das Tier nicht entkommen kann, aber wiederum leicht genug, dass der Pelz des Tiers nicht beschädigt wird. Den gefangenen Tieren werden durch die Fallen die Extremitäten gebrochen oder sie werden um den Hals oder Bauch in furchtbarer Lage festgehalten, bis die Fallensteller nach 1 bis 7 Tagen vorbeikommen. [...] $90 \%$ der in Fallen gefangenen Tiere gehören nicht zu der Tierart, für die die Fallen aufgestellt worden waren. Sie sind Abfall für die Pelzindustrie“ (Verein gegen Tierfabriken o. J. b).

„Ein Papst spricht“, kommentierte Seewald den Sachverhalt treffend, „immer auch in Gesten und Gebärden, Zeichen und Symbolen" (Benedikt XVI. 2010, 109). Offensichtlich hat Papst Benedikt XVI. die Symbolkraft des Pelztragens und seine Funktion als negatives Vorbild gewaltig unterschätzt. Italienische und internationale Tierschutzorganisationen forderten Benedikt auf, das Tragen von Pelz zu unterlassen, und sammelten Unterschriften für eine Internet-Petition an den Papst. Aus den eigenen Reihen erhielt der Papst vornehmlich Unterstützung. Ein altehrwürdiger Kardinal mit dem klingenden Namen Andrea Cordero Lanza di Montezemolo spielte die Proteste mit der klassisch tiervergessenen Argumentation der katholischen Moraltheologie herunter: „Gibt es nicht wichtigere Probleme?“, warf Eminenz ein. „Es gibt Menschen, die wesentlich dringender Hilfe brauchen, um die sich aber niemand sorgt. Und wenn wir Tiere essen, können wir sie auch anziehen“ (Don Kington 2008).

\section{Resümee: Der Karneval ist vorbei, Pelze sind out. Doch was ist mit Lederschuben?}

Papst Benedikts Vorliebe für luxuriöse Ästhetik und tiervergessene Schönheit fand bei seinem Nachfolger keinen Anklang. Eine höchstwahrscheinlich fiktive, aber vielsagende Geschichte berichtet davon, dass Papst Franziskus unmittelbar nach seiner Wahl in der Ankleidekammer neben der Sixtinischen Kapelle mit Papst Benedikts Zeremonienmeister Guido Marini aneinandergeraten sei. „,Das können Sie anziehen', soll der neue Papst 
den [!] verdutzten Geistlichen beschieden haben, als dieser ihn mit roter Mozetta und roten Schuhen ausstatten wollte. Und: ,Der Karneval ist vorbei““ (Daniel Deckers 2013).

Keine Pelze mehr bei Papst Franziskus, keine luxuriöse rote Fußbekleidung; unter dem Saum seiner Albe schauen schwarze Allerweltschuhe hervor. Gut so, aber nicht ganz. Es ist nämlich nicht anzunehmen, dass Franziskus Schuhe aus atmungsaktivem synthetischem Material trägt, von Hanf und Kork ganz zu schweigen. Dass er während seines Pontifikates noch einen Wechsel zu tierfreundlich produziertem Schuhwerk vollzieht, ist unwahrscheinlich.

In seiner Enzyklika Laudato si' spricht Papst Franziskus mit großem Respekt von Gottes Kreaturen. Er betont ihren intrinsischer Wert (Nrn. 16, 33, 69, 118, 208) und ihre Interdependenz (Nr. 89) und stellt ihnen einen Platz im himmlischen Jerusalem in Aussicht (Nr. 243). Einem „despotischen Anthropozentrismus" (Nr. 68), der die Kirchengeschichte jahrhundertelang dominierte, setzt der Papst die Überzeugung entgegen, dass Gottes lebenspendender Geist in allen Geschöpfen wohne (Nr. 88). Was die Tiere als Gottes Kreaturen betrifft, so stellt Franziskus eine enge Verbindung zwischen der Liebe zu Tieren und der Liebe zu Menschen her: „Das Herz ist nur eines, und die gleiche Erbärmlichkeit, die dazu führt, ein Tier zu misshandeln, zeigt sich unverzüglich auch in der Beziehung zu anderen Menschen. Jegliche Grausamkeit gegenüber irgendeinem Geschöpf ,widerspricht der Würde des Menschen“" (Nr. 92). Das sind hehre Worte und großartige Aussagen. Doch an ihnen wird auch ein grundlegendes Desiderat theologischer und kirchenamtlicher Tierethik sichtbar: Beide übersehen, dass die eigene Nahrung auf dem Teller, die eigenen Schuhe an den Füßen und die eigene Pelzmütze auf dem Kopf genau jene Produkte sind, die die theoretisch beklagte erbärmliche Misshandlung und Tötung tierischer Mitgeschöpfe praktisch voraussetzen.

Es hat in der menschlichen Geschichte Zeiten gegeben, in denen man sich anderer Tiere bedienen musste, um Kleidung herzustellen, und auch heute ist es in bestimmten geografischen Räumen wahrscheinlich noch unvermeidlich. An sich jedoch existieren gegenwärtig in vielen Regionen dieser Welt genügend Alternativen zu tierlichen Produkten:

„Es stehen viele Materialien zur Herstellung von Bekleidung zur Verfügung, die es nicht erfordern, andere Tiere dafür zu verwenden. [...] Christen [und andere Menschen guten Willens], die so leben wollen, dass ihre Mitgeschöpfe nicht aus Gründen der menschlichen Ernährung für sie getötet werden müssen, werden sich auch darum bemü- 
hen, Materialien für ihre Bekleidung auszusuchen, die ohne die Tötung von Tieren auskommen“ (David Clough 2019, 99-100).

\section{Literatur}

Albert Schweitzer Stiftung für unsere Mitwelt 2014 a, Leder: Tierleid und Umweltverschmutzung (veröffentlicht am 22.11.2014, zuletzt aktualisiert am 21.08.2016), in: https://albert-schweitzer-stiftung.de/aktuell/leder-tierleid-und-u mweltverschmutzung (abgerufen am 22.05.2020).

Albert Schweitzer Stiftung für unsere Mitwelt 2014 b, Daunen - immer wieder Lebendrupf (veröffentlicht am 22.02.2014, zuletzt aktualisiert am 31.08.2015), in: https://albert-schweitzer-stiftung.de/aktuell/daunen-lebendrupf (abgerufen am 22.05.2020).

Albert Schweitzer Stiftung für unsere Mitwelt 2016, Schafwolle: Tierschutz- und umweltrelevant (veröffentlicht am 24.11.2016, zuletzt aktualisiert am 13.02.2017); in: https://albert-schweitzer-stiftung.de/aktuell/schafwolle (abgerufen am 22.05.2020).

Allen, Charlotte 2013, Benedict XVI, the best-dressed pope. The pontiff dressed to reflect the beauty of faith, in: Los Angeles Times vom 17. Februar; online: http:// articles.latimes.com/2013/feb/17/opinion/la-oe-allen-pope-fashion-20130217 (abgerufen am 22.05.2020).

Arvay, Clemens G. 2016, Warum ich Pflanzen esse, aber keine Tiere, in: Vegan Magazin, August/September, 92-95.

Bailey, Sarah 2013, Clerical Vestments, Oxford/New York.

Benedikt XVI. 2010, Licht der Welt. Der Papst, die Kirche und die Zeichen der Zeit. Ein Gespräch mit Peter Seewald, Freiburg i. Br. etc.

Brophy, Brigid 1965, The Rights of Animals, in: The Sunday Times, Oktober, zitiert nach: https://cellulr.wordpress.com/2017/10/09/the-rights-of-animals/ (abgerufen am 22.05.2020).

Brophy, Brigid 1966, Unlived Life: A Manifesto Against Factory Farming. Hg. von Campaigners Against Factory Farming, zitiert nach: https://christianveg.org/thbrophy-brigid.htm (abgerufen am 22.05.2020).

Clough, David 2019, On Animals, Volume Two: Theological Ethics, London.

Coetzee, J[ohn] M[axwell] 1999, The Lives of Animals, Princeton.

Conway, Henry 2013, Pope Benedict: his true legacy is his fashion sense, in: The Guardian vom 3. März; online: https://www.theguardian.com/fashion/fashion-bl og/2013/mar/03/pope-benedict-true-legacy-fashion-sense (abgerufen am 22.05.2020).

Daunenarten o. J., in: https://www.daunendecke.de/daunenarten.html (abgerufen am 22.05.2020). 
Deckers, Daniel 2013, Der Karneval ist aus. Der Vatikan unter Papst Franziskus, in: Frankfurter Allgemeine Zeitung vom 24. März; online: https://www.faz.net/akt uell/politik/die-wahl-des-papstes/der-vatikan-unter-papst-franziskus-der-karnevalist-aus-12125804.html abgerufen am 22.05.2020).

Engström, Henrik/Coetzee, J[ohn] M[axwell] 2004, Animals, Humans, Cruelty and Literature: A Rare Interview with J. M. Coetzee, in: http://www.satyamag.com/ may04/coetzee.html (abgerufen am 22.05.2020).

Fabbretti, Nazareno 1990, Animals write ... Dear humans, Middlegreen (italienisch: Caro uomo. Lettere degli animali, Cinisello Balsamo 1988).

Foer, Jonathan Safran ${ }^{4} 2014$, Tiere essen, Frankfurt a. M.

Franziskus 2015, Laudato si'. Enzyklika über die Sorge für das gemeinsame Haus (24.05.2015).

Jolly, Jasper 2019, Dr Martens' profits up $70 \%$ with success of new „vegan“ range, in: The Guardian vom 12. August, in: https://www.theguardian.com/business/20 19/aug/12/dr-martens-profits-up-70-with-success-of-new-vegan-range (abgerufen am 22.05.2020).

Katechismus der Katholischen Kirche 2003. Neuübersetzung aufgrund der Editio typica latina von 1997, München etc.

Kington, Don 2008, Vatican: Pope makes fur fly over revival of ermine robes, in: The Guardian vom 14. August; online: https://www.theguardian.com/world/200 8/aug/14/catholicism.religion (abgerufen am 22.05.2020).

Philippi Collection 2010 a, Die Mozzetta des Papstes 2010, in: http:/www.dieter-ph ilippi.de/klerikale-feinheiten/die-mozzetta-des-papstes (abgerufen am 22.05.2020).

Philippi Collection 2010 b, Kopfbedeckungen, in: http://www.dieter-philippi.de/de /sammlung-philippi/kopfbedeckungen-1 (abgerufen am 22.05.2020).

ProTier - Stiftung für Tierschutz und Ethik o. J., Fakten zur Pelzproduktion, in: http:/www.protier.ch/site/index.cfm?id_art=97250\&actMenuItemID=45057\&vs prache $=$ de (abgerufen am 22.05.2020).

Remele, Kurt ${ }^{2}$ 2019, Die Würde des Tieres ist unantastbar. Eine zeitgemäße christliche Tierethik, Kevelaer.

Stella McCartney Takes On the Leather Trade 2015, in: https:/www.youtube.com/ watch? $\mathrm{v}=2 \mathrm{pY} 2 \mathrm{mATv} 26 \mathrm{E}$ (abgerufen am 22.05.2020).

Veblen, Thorstein [1899], The Theory of the Leisure Class, 33-47 (Chap. 4: Conspicuous Consumption), in: http://moglen.law.columbia.edu/LCS/theoryleisureclass.pdf (abgerufen am 22.05.2020).

Verein gegen Tierfabriken 2014, Daunen - ein Naturprodukt, in: https:/vgt.at/pres se/news/2014/news20141014es.php (abgerufen am 22.05.2020).

Verein gegen Tierfabriken o. J. a, Leder, in: https:/vgt.at/presse/news/2020/news20 200415ih.php (abgerufen am 22.05.2020).

Verein gegen Tierfabriken o. J. b, Hintergrundwissen Pelz, in: https://vgt.at/projekt e/pelz/fakten.php (abgerufen am 18.05.2020). 
Vier Pfoten 2020, Alternativen zu Mulesing, in: https://wollemitpo.vier-pfoten.at $/ \mathrm{m}$ ulesing-video (abgerufen am 22.05.2020).

Welt 2013, Tier-Haut-Couture: Pelze zwischen Mode und Moral (09.01.2013), in: http://www.welt.de/newsticker/leute/stars/article112636901/Tier-Haut-CouturePelze-zwischen-Mode-und-Moral.html (abgerufen am 22.05.2020). 
